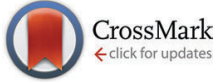

Cite this: Phys. Chem. Chem. Phys., 2017, 19, 1151

Received 23rd September 2016, Accepted 29th November 2016

DOI: $10.1039 / c 6 c p 06532 e$

www.rsc.org/pccp

\title{
Seeing the invisible plasma with transient phonons in cuprous oxide $\dagger$
}

\author{
Laszlo Frazer, ${ }^{\star a}$ Richard D. Schaller, ${ }^{\text {bc }}$ Kelvin B. Chang, ${ }^{c}$ Aleksandr Chernatynskiy ${ }^{d}$ \\ and Kenneth R. Poeppelmeier ${ }^{\text {ce }}$
}

\begin{abstract}
The emission of phonons from electron-hole plasma is the primary limit on the efficiency of photovoltaic devices operating above the bandgap. In cuprous oxide $\left(\mathrm{Cu}_{2} \mathrm{O}\right)$ there is no luminescence from electron-hole plasma. Therefore, we searched for optical phonons emitted by energetic charge carriers using phonon-to-exciton upconversion transitions. We found $14 \mathrm{meV}$ phonons with a lifetime of $0.916 \pm$ 0.008 ps and $79 \mathrm{meV}$ phonons that are longer lived and overrepresented. It is surprising that the higher energy phonon has a longer lifetime.
\end{abstract}

\section{Introduction}

Cuprous oxide is a semiconductor with a high theoretical photovoltaic/photocatalytic efficiency, composed of abundant and nontoxic elements. ${ }^{1,2}$ The well-known Shockley-Queisser efficiency limit for a single junction solar cell assumes that all above-gap photons generate the same voltage owing to rapid thermalisation. ${ }^{3}$ Recently, first principles calculations have shown that the energy above the bandgap is lost primarily to phonon radiation and secondarily to charge carrier interactions. ${ }^{4}$ Here, we use time-resolved phonon-to-exciton ${ }^{5,6}$ transitions to detect phonon radiation from charge carriers.

Charge carrier/phonon inelastic scattering can be examined by measuring carriers or by measuring phonons. For example, the depletion of energy from electron-hole plasma has been measured using time resolved luminescence in several materials. ${ }^{7-12}$ However, in cuprous oxide, both the $3 \mathrm{~d}$-like valence and the $4 \mathrm{~s}^{-l i k e} \mathrm{e}^{13}$ conduction band have positive parity. Therefore, electron-hole plasma luminescence is forbidden and not observed. Plasma relaxation can be measured with surface electron photoemission ${ }^{14,15}$ and

\footnotetext{
${ }^{a}$ School of Chemistry, UNSW Sydney, NSW 2052, Australia and Department of Chemistry, Temple University, 1901 N. 13th Street, Philadelphia, PA 19122, USA. E-mail: pccp@laszlofrazer.com; Tel: +1 61401648058

${ }^{b}$ Center for Nanoscale Materials, Argonne National Laboratory, 9700 South Cass Avenue, Building 440, Argonne, IL 60439, USA

${ }^{c}$ Department of Chemistry, Northwestern University, 2145 Sheridan Road, Evanston, Illinois 60208-3112, USA

${ }^{d}$ Department of Physics, Missouri University of Science and Technology, 117 Physics Building, Rolla, Missouri 65409-0640, USA

${ }^{e}$ Chemical Sciences and Engineering Division, Argonne National Laboratory, 9700 South Cass Avenue, Argonne, Illinois 60439, USA

$\dagger$ Electronic supplementary information (ESI) available: One figure providing a graphical breakdown of the analysis of transient absorption in cuprous oxide and a document describing the amplitudes of the model components and tests of the standard error calculations. See DOI: 10.1039/c6cp06532e
}

nanosecond scale cyclotron resonance. ${ }^{16}$ This led us to develop a bulk, femtosecond scale phonon measurement approach.

\section{Experimental}

\subsection{Transient absorption}

Transient absorption has been used to measure hot carrier lifetimes in bulk crystals of $\mathrm{MoS}_{2}(50 \mathrm{ps})^{17}$ and to detect hot carriers in bulk crystals of $\alpha-\mathrm{Fe}_{2} \mathrm{O}_{3}{ }^{18}$ It has also been applied to electron-hole recombination in bulk, ${ }^{19}$ which is a forbidden process in cuprous oxide. In cuprous oxide, mid-infrared transient absorption has been used for excitonic Lyman spectroscopy. ${ }^{20}$ Electron beam excitation, as opposed to optical excitation, has also been used. ${ }^{21}$ There are also numerous studies of nanomaterials such as ref. 22 . To the best of our knowledge, this is the first report of time resolved measurements of phonon-to-exciton transitions.

Here, we report the use of a pump pulse to produce an optically dark electron-hole plasma $(|1\rangle)$ that results in emission of phonons by the carriers $(|2\rangle)$. The carriers in the plasma may relax through a variety of bands. ${ }^{23}$ The absorbance is then measured to obtain the dynamics of the two phonon modes $(|3\rangle)$. These three steps are illustrated in Fig. 1.

We used a regeneratively amplified 35 fs Ti:Sapphire laser. The amplifier output was split into pump and probe beams. The pump beam passed through a delay line. It was frequency doubled to $400 \mathrm{~nm}$ in a $\beta$-barium borate crystal. The fundamental was filtered out. The pump was chopped from $2 \mathrm{kHz}$ to a $1 \mathrm{kHz}$ repetition rate. $22 \mathrm{~J} \mathrm{~m}^{-2}$ were focused on to the sample in a spot with a diameter of $0.85 \mathrm{~mm}$ to $1 / e$.

This pump configuration can access transitions up $2.62 \mathrm{eV}$ to the second conduction band that are dipole allowed and that lie above the four exciton series. ${ }^{23}$ The conduction bands near the surface were pumped with about $10^{32}$ electrons $\mathrm{s}^{-1} \mathrm{~m}^{-2}$ at 


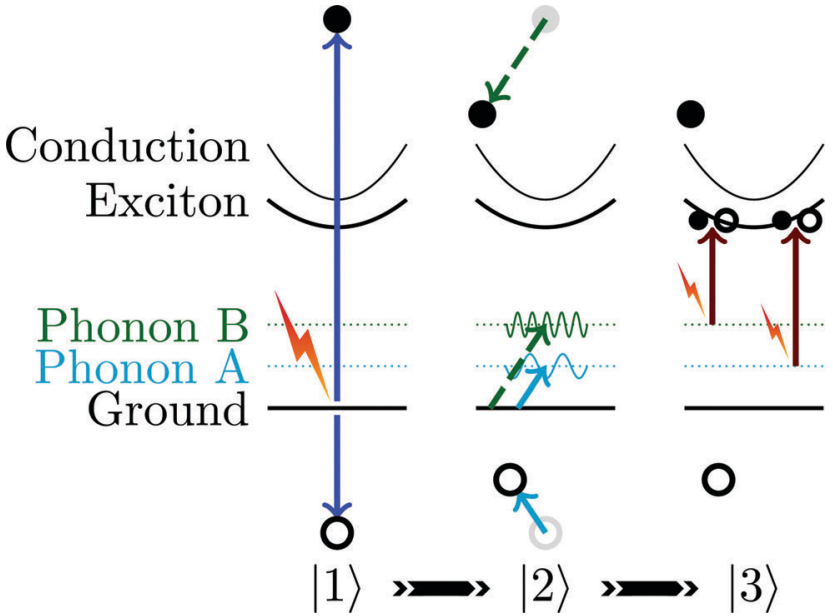

Fig. 1 In this cartoon, a pump laser pulse excites electrons and holes $|1\rangle$. Then the carriers relax by emission of various phonons $|2\rangle$. The probe measures transient absorption from phonon states to exciton states $|3\rangle$.

the peak of each laser pulse. The peak pump irradiance is $10^{12}$ times typical sunlight irradiance. ${ }^{24}$ While the rate of nonthermal phonon upconversion to excitons is small in a typical cuprous oxide solar cell when compared to this experiment, the bath of thermal optical phonons is much larger. At ambient temperatures, about $100 \mathrm{meV}$ of sub-bandgap absorption, which is driven by thermal phonon upconversion, contributes to the efficiency of the solar cell, assuming a thickness of $1 \mathrm{~mm}$ or more. ${ }^{6}$

The probe beam was converted to white light using selfphase-matching in a $2 \mathrm{~mm}$ thick sapphire. The fundamental was filtered out. The beam was tightly focused on the sample at a small angle to the pump beam and in the centre of the pump beam.

The cuprous oxide crystal was grown using our previously reported method for producing relatively large, phase-pure crystals with minimal point defects. ${ }^{25-28}$ For this experiment, the sample with [100] faces from ref. 29 was mounted on a sapphire in vacuum at a temperature of $3.2 \mathrm{~K}$ to reduce the thermal background. ${ }^{6,30}$ The crystal was oriented with the pump beam travelling in the [100] direction, which is normal to the surface. The pump beam's electric field was polarised parallel to [010].

The transmitted portion of the probe beam was collected in an Ultrafast Systems Helios spectrometer with $2 \mathrm{~nm}$ resolution. The resolution is not sufficient to observe the $1 \mathrm{~s}$ orthoexciton polariton. Probe shots with and without the pump beam were recorded for various positions of the pump delay line to determine the time dependence of the phonon relaxation. The baseline measured when the probe arrived before the pump was subtracted from the data.

\subsection{Ab initio methods}

To better understand the measurements, we also performed firstprinciples calculations of the phonon lifetimes at the $\Gamma$-point. The band extrema are located at the $\Gamma$-point. Our phonon lifetime calculations assume anharmonicity as a perturbation to the harmonic problem; for the low temperatures considered in our experiments, this is well-obeyed approximation. This is further supported by the experimental lack of interaction between transient phonons in Fig. 4 as discussed below. Theoretical details in the context of inelastic neutron scattering can be found in ref. 31. Computations were performed using the software package PhonTS ${ }^{32}$ developed by one of the authors. Second and third order force constants necessary for this calculation were obtained from standard density functional calculations (DFT) within the local density approximation (LDA) using the finite displacement method. A supercell consisting of $2 \times 2 \times 2$ primitive cells was used for the calculations with a $500 \mathrm{eV}$ energy cutoff for the plainwave expansion of the electronic wavefunction and $\Gamma$-point sampling of the Brillouin zone. Past calculations have shown that anharmonic terms are relatively short ranged in semiconductors. Larger supercells are not required for these calculations. ${ }^{33,34}$ Calculations were performed with the Vienna Ab Initio Simulation Package (VASP). ${ }^{35-38}$ While hybrid functionals are required in order to obtain accurate electronic structure, ${ }^{39}$ phonon properties are sufficiently accurate at the LDA level. ${ }^{40}$

\section{Results and discussion}

\subsection{Transient absorption analysis}

3.1.1 Modelling of backgrounds. Instead of correcting chirp, we included a linear probe chirp in our global analysis model. With our approach, the starting time of the signal is wavelength dependent. We modelled the absorption with a function including a $\chi^{(3)}$ based coherent artefact. This component of the model, which was taken from ref. 41 and 42 , is shown in ESI, $\dagger$ Fig. S1(a). We also found a background that we approximated with a wavelengthindependent transient with lifetime $0.736 \pm 0.003 \mathrm{ps}$, shown in ESI, $\dagger$ Fig. S1(b). The wavelength-independence of ESI, $\dagger$ Fig. S1(g) from 640 to $675 \mathrm{~nm}$ is experimental support for this approximation.

3.1.2 Literature assignment of spectra in cuprous oxide. The cuprous oxide lattice has the highest possible symmetry (order 48). It consists of an oxygen body centred cubic lattice with each oxygen atom tetrahedrally coordinated to four copper atoms. The tetrahedra share all four corners forming straight $180^{\circ} \mathrm{O}-\mathrm{Cu}-\mathrm{O}$ bond angles. The A phonon is ${ }^{2} \Gamma_{12}{ }^{-}$and has energy $14 \mathrm{meV}$. It corresponds to compression of opposing edges of the tetrahedra. The B phonon is ${ }^{3} \Gamma_{15}{ }^{-}$and has energy $79 \mathrm{meV}$. It corresponds to transverse relative motion of the copper and oxygen lattices. ${ }^{43,44}$

We found that changes in the number of optical phonons present can be measured using so-called phonon to 1s orthoexciton transitions. ${ }^{6,45}$ The exciton series in question is called the 'yellow' series because it is the lowest lying set of electronhole bound states. ${ }^{23}$ Since the phonon density of states is spectrally sharp, ${ }^{46,47}$ the structure of the transient absorbance $A(E, t)$ as a function of energy depends on the phonon energy and the exciton density of states. According to Fermi's golden rule, ${ }^{6}$

$$
A(E, t)=\sum_{\mathrm{p} \in\{\text { phonons }\}} a_{\mathrm{p}} n_{\mathrm{p}}(t) \sqrt{E-\left(E_{\mathrm{X}}-E_{\mathrm{p}}\right)}
$$


where $a_{\mathrm{p}}$ determines the relative efficiency of the absorption of the modes, $n_{\mathrm{p}}(t)$ is the transient occupation of that mode at time $t$ after light absorption, $E_{\mathrm{X}}$ is the orthoexciton ground state energy, and $E_{\mathrm{p}}$ is the energy ${ }^{45}$ of a particular phonon mode p. $E_{\mathrm{X}}$ and $E_{\mathrm{p}}$ were assumed.

3.1.3 Phonon dynamics. The dynamics of $n_{\mathrm{p}}(t)$ are governed by a pump term (assuming a Gaussian profile with amplitude $C$ and duration $\sigma$ ) and a decay term (with lifetime $\tau_{\mathrm{p}}$ ). Our results are insensitive to the temporal structure of the pump model.

$$
\frac{\mathrm{d}}{\mathrm{d} t} n_{\mathrm{p}}(t)=C \mathrm{e}^{-\frac{t^{2}}{2 \sigma^{2}}}-\frac{n_{\mathrm{p}}(t)}{\tau_{\mathrm{p}}}
$$

From 113894 data points (Fig. 2(a)), we found that $\tau_{\mathrm{A}}=0.916 \pm$ $0.008 \mathrm{ps}$ and $\tau_{\mathrm{B}}=31 \pm 3 \mathrm{ps}$. The uncertainties account for random errors only. Validation of the error calculations is described in the ESI. $\dagger$ In other materials, phonon lifetimes are typically in the few picosecond range. ${ }^{48,49}$ Transitions involving additional phonons are not expected to be observed based on previous experimental results. ${ }^{6}$ Transition matrix elements for the various phonons that have not yet been observed participating in phonon-to-exciton transitions are an interesting area for potential future investigation.

Fig. S1(c) and (d) (ESI $\dagger$ ) show the models of each of the phonon assisted transitions used to determine $n_{\mathrm{p}}(t)$. For these

\section{(a)}

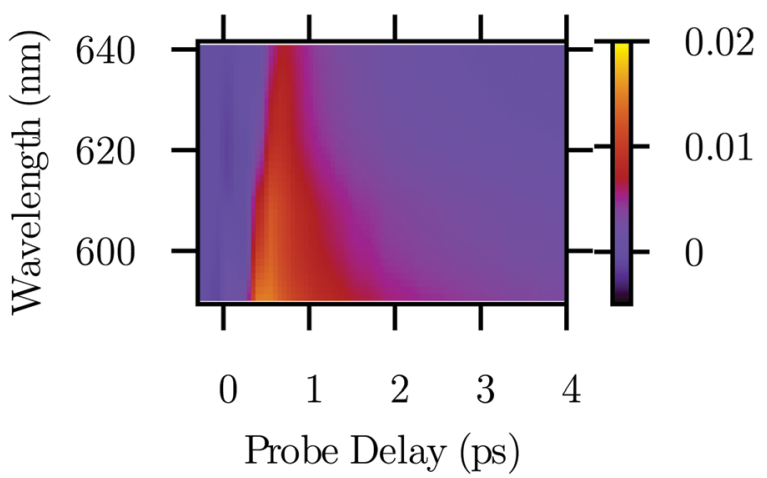

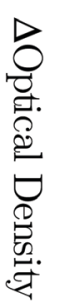

(b)

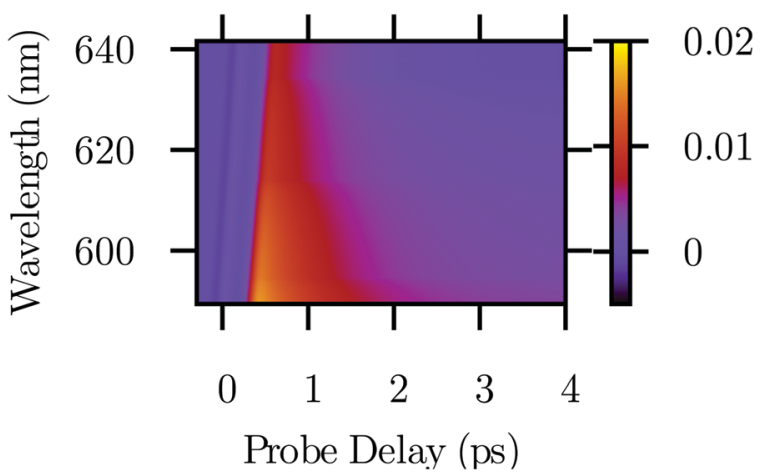

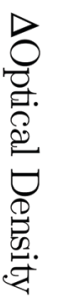

Fig. 2 (a) Transient absorption, baseline subtracted and down sampled to a grid with a Gaussian kernel. (b) The complete model of the transient absorption data including our models of phonon production and phononto-exciton absorption transitions. The model (b) is intended to resemble the data (a). The model (b) is detailed in Section 3.1 and in the ESI. $\dagger$ model parts, only the amplitude $a_{\mathrm{p}} n_{\mathrm{p}}(0)$ and lifetime $\tau_{\mathrm{p}}$ were independent regression parameters; literature phonon and exciton energies were assumed because they are more precise. ${ }^{30,50}$ Ref. 6 , Fig. 4 provides particularly elegant time averaged data validating the spectral assignments we are reusing. The phonon spectrum is consistent between neutron, Raman, absorption, luminescence, and density functional theory methodologies. ${ }^{46}$

Above $2.085 \mathrm{eV}$ (595 nm), we serendipitously found a feature corresponding to a superposition of phonon-to-exciton transitions with the exciton $n>1 .{ }^{13,51}$ It has a lifetime of $9.8 \pm 0.5 \mathrm{ps}$ and the same amplitude as the $\mathrm{B}$ to $1 \mathrm{~s}$ transition. This portion of our model, which is treated mathematically the same way as processes A and B for simplicity, is shown in ESI, $\dagger$ Fig. S1(e). Above $2.15 \mathrm{eV}$, not enough light was transmitted to allow for measurements of transient absorption.

The total model, including time and wavelength dependence of the transient absorption, is shown in Fig. 2(b) to agree with the data in Fig. 2(a). A broader view of the complete model (f) and data $(\mathrm{g})$ is shown in ESI, $\dagger$ Fig. S1. We excluded data for wavelengths greater than $640 \mathrm{~nm}$ from the analysis because they do not include the signals of interest.

\subsection{Partition of energy into phonon modes: relative amplitudes of phonon state occupation}

Based on the equipartition theorem, the steady state occupation of A should be about six times that of $\mathrm{B}$. This is consistent with the relative strengths of the steady state A/B phonon-to-exciton absorption features. ${ }^{6,52,53}$ We conclude $a_{\mathrm{A}} \approx a_{\mathrm{B}}$. In the transient absorption spectrum, however, $a_{\mathrm{A}} n_{\mathrm{A}}(0) /\left(a_{\mathrm{B}} n_{\mathrm{B}}(0)\right)=2.0 \pm 0.3 \ll 6$, indicating that carriers release about a third as much energy in the form of phonon A as in the form of phonon B. Future electron-phonon scattering calculations ${ }^{4,54-57}$ may explain the distribution of emitted phonons and the phonon emission and absorption rates. ${ }^{57,58}$

\subsection{Power dependence}

In order to test for density-dependent effects, we studied the dependence of the transient absorption on the excitation power. We did not observe any density-dependent effects, which might have included absorption of phonons by electron-hole plasma. The absorption dynamics at $600 \mathrm{~nm}$ for three laser excitation powers are shown in Fig. 3. This wavelength includes both the $\mathrm{A}$ and $\mathrm{B}$ phonon assisted transient absorption. A single exponential representing $\tau_{\mathrm{A}}$ is drawn in solid black to show that the data are not a single exponential decay. The data are in reasonable agreement with the hypothesis that the phonon lifetimes are independent of the laser excitation power (Fig. 3 lines). There may be a dip in the lower power data around 1 ps.

The creation of phonons is illustrated in Fig. 6(a). Phonon absorption is illustrated in Fig. 6(f). In our conditions, the phonon density is driven by conduction carriers, which create phonons, and phonon relaxation, as opposed to phonon absorption by conduction carriers, exciton-exciton annihilation, or phonon-phonon annihilation. The rates of these three excluded processes are dependent on the excitation power, but no power dependence is observed in Fig. 3. The excluded 


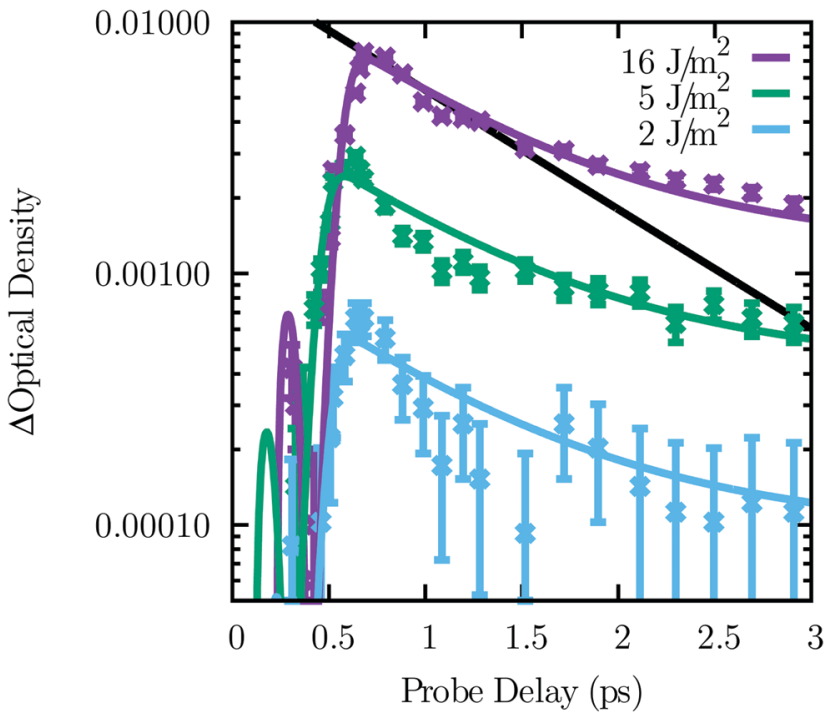

Fig. 3 Example transient absorption dynamics at $600 \mathrm{~nm}$ for three incident pump radiant exposures (colours) with the model (dashed lines). A single exponential representing $\tau_{\mathrm{A}}$ is drawn in solid black to show that the data are not a single exponential decay. $0.02 \%$ of the time and wavelength resolved data used in the analysis is displayed.

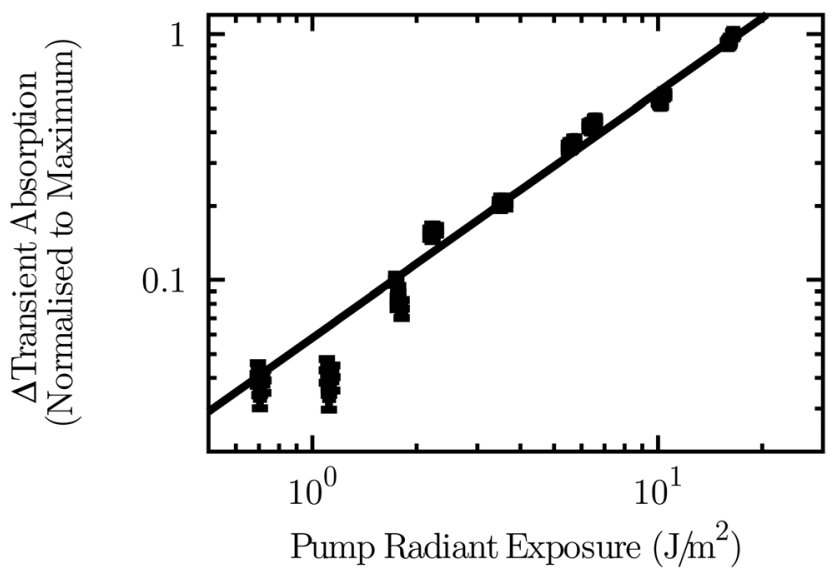

Fig. 4 Dependence of transient absorption at $600 \mathrm{~nm}$ on the incident pump radiant exposure.

processes are power dependent because at low temperature they can only occur when two photoexcited states interact. In a highly doped semiconductor ${ }^{55,56,58,59}$ or a metal, ${ }^{57}$ absorption of phonons by carriers would dominate, even in the absence of photoinduced carriers.

The phonon density should grow linearly with pump intensity. The amplitude of the transient absorption was determined versus laser power. All components of the model were assumed to scale proportionately. The linear relationship is confirmed in Fig. 4.

\subsection{Origin of transient phonons: short-lived plasma and long- lived excitons}

By selecting a high excitation energy $(3 \mathrm{eV})$, we have caused the intraband and second-to-first conduction (or valence) band transitions to be the primary source of phonons. Of these four bands, only the second conduction band has negative parity, suggesting it plays an important role in the emission of negative parity phonons. Transitions to and between the exciton states in the four exciton series ${ }^{13,23,60,61}$ also contribute to phonon production. The energy breakdown of $3 \mathrm{eV}$ is $30 \%$ conduction carrier relaxation (Fig. 6(a)), 5\% yellow exciton formation/thermalisation (Fig. 6(e)), and 66\% bandgap.

Owing to the high density of states, conduction carriers primarily relax within the timescales studied here. Conduction carrier relaxation times are typically shorter than phonon lifetimes. This is the basis for hot phonon engineering. ${ }^{7,62}$ The carrier relaxation includes electron-hole annihilation and exciton formation that reduces the carrier number in a manner that is strongly density-dependent. In the future, in order to introduce additional phonon scattering processes, heavy doping could be used to add conduction carriers or higher temperatures could be used to add bath phonons. These potential future experiments will lead to additional complexity in the absorption spectrum. ${ }^{6,52,53}$

The rise time of the phonons caused by phonon emission from high energy electron-hole plasma is not observed nor do we expect it to be resolvable with currently available shortpulsed lasers. The complex process of exciton thermalisation, however, relies on phonon emission through strict selection rules. ${ }^{63}$ The thermalisation time can exceed the exciton lifetime at low temperatures. ${ }^{64}$ These slow phonon emission processes were not the target of this study. We expect they are too weak and too slow to contribute meaningfully to the data, which we interpret in terms of phonon lifetimes, not phonon excitation rates.

Exciton thermalisation by phonon emission, and its manipulation by strain, are crucial to exciton relaxation explosion ${ }^{63,64}$ and exciton/vacancy binding. ${ }^{65}$ All these processes can be repumped by inelastic exciton-exciton scattering. ${ }^{16,66-69}$

\subsection{Potential additional backgrounds}

Our interest is in the initial electron-hole plasma. We do not extrapolate the results beyond the 4 ps time window of the 113894 data points shown in Fig. 2. The signal to noise ratio for a single data point peaks at around 200 .

Owing to two kinds of known background, we did not collect data beyond 4 ps for the analysis of phonon-to-exciton transitions. First, some of the exciton relaxation routes are slow; ${ }^{70}$ as a result $n_{\mathrm{p}}$ was detectably greater than zero at the longest delay we had available (6 ns). Second, as shown in Fig. 5, there are oscillations in the transient absorption consistent with the known $43 \mathrm{GHz}$ Brillouin mode of cuprous oxide. ${ }^{71,72}$ Similar coherent phonon oscillations, whose frequencies have a slight wavelength dependence arising from refractive index variation, have been observed in other materials. ${ }^{73-76}$ These two backgrounds contribute to the systematic error in the model. They are smaller than the phonon-to-exciton transition signal originating from the electron-hole plasma.

The electron-hole plasma may absorb the probe light. This process gets stronger at longer wavelengths and has been observed in the infrared in other materials. ${ }^{77}$ We did not find any evidence for this phenomenon. 


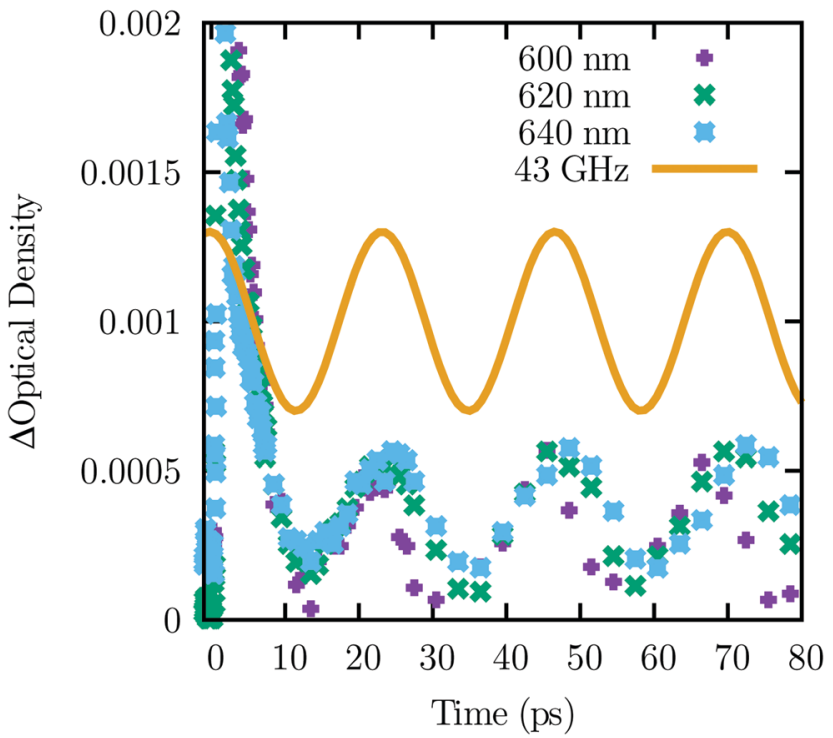

Fig. 5 Low frequency phonons cause oscillations in the transient absorption. The curve shows the Brillouin frequency ${ }^{71,72}$ as a guide to the eye. The range of the initial transient is not shown.

\subsection{Ab initio results}

Our results for phonon frequencies are in good agreement with previous results, which have also shown the density of states. ${ }^{46}$ Computed lifetimes for all phonons at the $\Gamma$-point are presented in Table 1. The A phonon has a lifetime of $1.98 \mathrm{ps,} \mathrm{in} \mathrm{good} \mathrm{agreement}$ with experimental value. Interestingly, the ${ }^{3} \Gamma_{25}{ }^{+}$phonon, which does not induce absorption, ${ }^{6,45}$ has a very long lifetime of 178 ps.

The B phonon, however, has a much shorter calculated lifetime than measured lifetime. The disagreement suggests that the $\mathrm{B}$ phonon occupation is selectively repumped. One well-known mechanism of repumping from cold exciton states to hot carrier states is Auger recombination. Auger recombination is a densitydependent process, but the repumping of conduction carriers may or may not have a density dependent rate. ${ }^{78}$ It depends on the relative magnitude of density-dependent and density-independent decay of the underlying exciton states. Different measurements of Auger recombination are reviewed in ref. 79 and 80.

We note that B (but not A) phonons may be produced by potentially slow relaxation of carriers from about $54 \mathrm{meV}$ above the bandgap to $2 \mathrm{p}$ excitons (binding energy $25 \mathrm{meV}$ ). We did not find any evidence for multiple timescales in the B signal, so if transitions to $2 \mathrm{p}$ excitons explain $\tau_{\mathrm{B}}$ then they are the primary

Table 1 Computed phonon energies and lifetimes in $\mathrm{Cu}_{2} \mathrm{O}$ at the $\Gamma$-point

\begin{tabular}{lccc}
\hline Phonon & $E_{\mathrm{p}}(\mathrm{meV})$ & Computed $\tau_{\mathrm{p}}(\mathrm{ps})$ & Measured $\tau_{\mathrm{p}}(\mathrm{ps})$ \\
\hline${ }^{3} \Gamma_{25}{ }^{-}$ & 8.4 & 23.8 & \\
${ }^{2} \Gamma_{12}{ }^{-}(\mathrm{A})$ & 9.5 & 1.98 & $0.916 \pm 0.008$ \\
${ }^{3} \Gamma_{15}{ }^{-}$ & 18.8 & 1.68 & \\
${ }^{3} \Gamma_{15}{ }^{-}$ & 20.0 & 1.11 & \\
${ }^{1} \Gamma_{2}{ }^{-}$ & 41.1 & 15.0 & $31 \pm 3$ \\
${ }^{3} \Gamma_{25}{ }^{+}$ & 62.5 & 178 & \\
${ }^{3} \Gamma_{15}{ }^{-}(\mathrm{B})$ & 75.8 & 0.53 & \\
${ }^{3} \Gamma_{15}{ }^{-}$ & 78.1 & 0.69 &
\end{tabular}

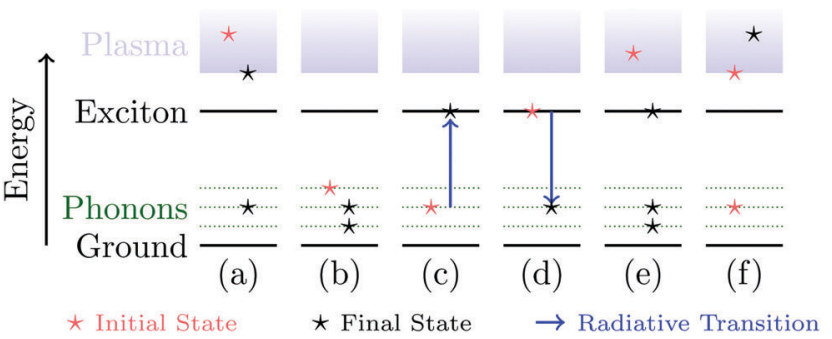

Fig. 6 Simplified energy level diagrams for six different phonon scattering processes, with the initial and final states indicated. Process (a): emission of a phonon by the plasma of conduction carriers, which we used to set up our experiment. Process (b): relaxation of a phonon to lower energy phonon states, which is our interpretation of the lifetimes reported here. Process (c): phonon-to-exciton transitions used to perform the measurements. Process (d): phonon-assisted luminescence, which is well known. ${ }^{27,50}$ Process (e): exciton formation, which is in need of investigation and cannot be distinguished from process (a) in this experiment. Process (f): excitation of conduction carriers by a phonon. Process ( $f$ ) is important at high carrier concentrations. Varying the excitation intensity to change the carrier concentration in the electron-hole plasma did not produce a detectable change in the dynamics, so we interpret our results in terms of process (b) rather than process (f).

source of B phonons. This concept could be tested by measuring bleach of the $2 \mathrm{p}$ line in a thinner sample. ${ }^{13}$

\section{Conclusions}

We determined the lifetime and relative occupation of two phonon modes in the first picosecond after light absorption. These phonons were detected using absorptive transitions from the phonon state to the 1s orthoexciton state (Fig. 6(c)). Prior knowledge of the phonon and exciton density of states provides for assignment of the absorption bands.,.$^{6,50,52,53}$ The higher energy mode has a surprisingly long lifetime.

The phonons we measured originate from relaxation of free carriers towards the conduction band minimum (Fig. 6(a)). This relaxation process is an important factor that caps the high theoretical solar energy conversion efficiency of cuprous oxide, a high elemental abundance, nontoxic semiconductor. ${ }^{1,2}$ Phonon emission can remove all charge carrier energy that is above the bandgap. We have captured phononic waste heat and light that is usually transmitted. We stored the energy in excitons. If these additional excitons ${ }^{6}$ can be utilised in future photovoltaic devices, ${ }^{5}$ it will increase the efficiency of solar energy conversion.

\section{Acknowledgements}

L. F. acknowledges NSF DGE-0801685 and the Institute for Sustainability and Energy at Northwestern. Use of the Center for Nanoscale Materials was supported by the U.S. Department of Energy, Office of Science, Office of Basic Energy Sciences, under Contract No. DE-AC02-06CH11357. Crystal growth was supported by NSF DMR-1307698 and in part by Argonne National Laboratory under U.S. Department of Energy contract DE-AC36-08GO28308. We acknowledge the X-ray and OMM 
Facilities at the MRC of Northwestern, which are supported by NSF MRSEC program DMR-1121262.

\section{References}

1 L. Y. Isseroff and E. A. Carter, Chem. Mater., 2013, 25, 253-265.

2 W. Septina, S. Ikeda, M. A. Khan, T. Hirai, T. Harada, M. Matsumura and L. M. Peter, Electrochim. Acta, 2011, 56, 4882-4888.

3 W. Shockley and H. J. Queisser, J. Appl. Phys., 1961, 32, 510-519.

4 M. Bernardi, D. Vigil-Fowler, J. Lischner, J. B. Neaton and S. G. Louie, Phys. Rev. Lett., 2014, 112, 257402.

5 J. R. Santos, M. I. Vasilevskiy and S. A. Filonovich, Phys. Rev. B: Condens. Matter Mater. Phys., 2008, 78, 245422.

6 P. W. Baumeister, Phys. Rev., 1961, 121, 359.

7 Y. Zhang, M. J. Tayebjee, S. Smyth, M. Dvořák, X. Wen, H. Xia, M. Heilmann, Y. Liao, Z. Zhang and T. Williamson, et al., Appl. Phys. Lett., 2016, 108, 131904.

8 R. Clady, M. J. Tayebjee, P. Aliberti, D. König, N. J. EkinsDaukes, G. J. Conibeer, T. W. Schmidt and M. A. Green, Prog. Photovoltaics, 2012, 20, 82-92.

9 L. Hirst, M. Führer, D. J. Farrell, A. LeBris, J.-F. Guillemoles, M. J. Tayebjee, R. Clady, T. W. Schmidt, Y. Wang and M. Sugiyama, et al., IEEE Photovoltaic Spec. Conf., 34th, 2011, 003302.

10 J. Ryan, R. Taylor, A. Turberfield, A. Maciel, J. Worlock, A. Gossard and W. Wiegmann, Phys. Rev. Lett., 1984, 53, 1841.

11 T. Elsaesser, J. Shah, L. Rota and P. Lugli, Phys. Rev. Lett., 1991, 66, 1757.

12 H. Yoshida, H. Saito, S. Shionoya and V. Timofeev, Solid State Commun., 1980, 33, 161-165.

13 T. Kazimierczuk, D. Fröhlich, S. Scheel, H. Stolz and M. Bayer, Nature, 2014, 514, 343-347.

14 T. Rohwer, S. Hellmann, M. Wiesenmayer, C. Sohrt, A. Stange, B. Slomski, A. Carr, Y. Liu, L. M. Avila and M. Kalläne, et al., Nature, 2011, 471, 490-493.

15 J.-C. Deinert, D. Wegkamp, M. Meyer, C. Richter, M. Wolf and J. Stähler, Phys. Rev. Lett., 2014, 113, 057602.

16 N. Naka, I. Akimoto, M. Shirai and K. Kan'no, Phys. Rev. B: Condens. Matter Mater. Phys., 2012, 85, 035209.

17 N. Kumar, J. He, D. He, Y. Wang and H. Zhao, J. Appl. Phys., 2013, 113, 133702 .

18 A. G. Joly, J. R. Williams, S. A. Chambers, G. Xiong, W. P. Hess and D. M. Laman, J. Appl. Phys., 2006, 99, 053521.

19 R. Katoh, M. Murai and A. Furube, Chem. Phys. Lett., 2008, 461, 238-241.

20 T. Tayagaki, A. Mysyrowicz and M. Kuwata-Gonokami, J. Phys. Soc. Jpn., 2005, 74, 1423-1426.

21 R. Fuller, R. Williams and M. Kabler, Phys. Rev. Lett., 1970, 25, 446.

22 M. A. Mahmoud, W. Qian and M. A. El-Sayed, Nano Lett., 2011, 11, 3285-3289.

23 J. Schmutzler, D. Fröhlich and M. Bayer, Phys. Rev. B: Condens. Matter Mater. Phys., 2013, 87, 245202.
24 R. C. Temps and K. Coulson, Sol. Energy, 1977, 19, 179-184. 25 K. B. Chang, L. Frazer, J. J. Schwartz, J. B. Ketterson and K. R. Poeppelmeier, Cryst. Growth Des., 2013, 13, 4914-4922.

26 L. Frazer, K. B. Chang, K. R. Poeppelmeier and J. B. Ketterson, Sci. Technol. Adv. Mater., 2015, 16, 034901.

27 L. Frazer, E. J. Lenferink, K. B. Chang, K. R. Poeppelmeier, N. P. Stern and J. B. Ketterson, J. Lumin., 2015, 159, 294-302.

28 L. Frazer, K. B. Chang, R. D. Schaller, K. R. Poeppelmeier and J. B. Ketterson, J. Lumin., 2017, 183, 281-290.

29 L. Frazer, K. B. Chang, K. R. Poeppelmeier and J. B. Ketterson, Phys. Rev. B: Condens. Matter Mater. Phys., 2014, 89, 245203.

30 T. Ito and T. Masumi, J. Phys. Soc. Jpn., 1997, 66, 2185-2193.

31 A. A. Maradudin and A. E. Fein, Phys. Rev., 1962, 128, 2589-2608.

32 A. Chernatynskiy and S. R. Phillpot, Comput. Phys. Commun., 2015, 192, 196-204.

33 L. Lindsay, D. Broido and T. Reinecke, Phys. Rev. B: Condens. Matter Mater. Phys., 2013, 87, 165201.

34 A. Chernatynskiy, J. E. Turney, A. J. McGaughey, C. H. Amon and S. R. Phillpot, J. Am. Ceram. Soc., 2011, 94, 3523-3531.

35 G. Kresse and J. Hafner, Phys. Rev. B: Condens. Matter Mater. Phys., 1993, 47, 558-561.

36 G. Kresse and J. Hafner, Phys. Rev. B: Condens. Matter Mater. Phys., 1994, 49, 14251-14269.

37 G. Kresse and J. Furthmüller, Phys. Rev. B: Condens. Matter Mater. Phys., 1996, 54, 11169-11186.

38 G. Kresse and J. Furthmüller, Comput. Mater. Sci., 1996, 6, 15-50.

39 L. Y. Isseroff and E. A. Carter, Phys. Rev. B: Condens. Matter Mater. Phys., 2012, 85, 235142.

40 K.-P. Bohnen, R. Heid, L. Pintschovius, A. Soon and C. Stampfl, Phys. Rev. B: Condens. Matter Mater. Phys., 2009, 80, 134304.

41 B. Dietzek, T. Pascher, V. Sundström and A. Yartsev, Laser Phys. Lett., 2007, 4, 38.

42 S. Kovalenko, A. Dobryakov, J. Ruthmann and N. Ernsting, Phys. Rev. A: At., Mol., Opt. Phys., 1999, 59, 2369.

43 R. Elliott, Phys. Rev., 1961, 124, 340.

44 K. O'Hara, PhD thesis, University of Illinois at UrbanaChampaign, 1999.

45 P. Y. Yu and Y. Shen, Phys. Rev. B: Condens. Matter Mater. Phys., 1975, 12, 1377.

46 P. A. Korzhavyi and B. Johansson, Literature review on the properties of cuprous oxide $\mathrm{Cu}_{2} \mathrm{O}$ and the process of copper oxidation, Swedish Nuclear Fuel and Waste Management Company, 2011.

47 M. Beg and S. Shapiro, Phys. Rev. B: Condens. Matter Mater. Phys., 1976, 13, 1728.

48 R. Alfano and S. Shapiro, Phys. Rev. Lett., 1971, 26, 1247.

49 F. Vallee, Phys. Rev. B: Condens. Matter Mater. Phys., 1994, 49, 2460.

50 Y. Petroff, P. Yu and Y. Shen, Phys. Rev. B: Condens. Matter Mater. Phys., 1975, 12, 2488.

51 C. Uihlein, D. Fröhlich and R. Kenklies, Phys. Rev. B: Condens. Matter Mater. Phys., 1981, 23, 2731.

52 T. Itoh and S.-I. Narita, J. Phys. Soc. Jpn., 1975, 39, 140-147. 53 T. Itoh and S.-I. Narita, J. Phys. Soc. Jpn., 1975, 39, 132-139. 
54 H. Kawai, G. Giorgi, A. Marini and K. Yamashita, Nano Lett., 2015, 15, 3103-3108.

55 B. Liao, J. Zhou, B. Qiu, M. S. Dresselhaus and G. Chen, Phys. Rev. B: Condens. Matter Mater. Phys., 2015, 91, 235419.

56 N. Tandon, J. Albrecht and L. Ram-Mohan, J. Appl. Phys., 2015, 118, 045713.

57 Y. Wang, Z. Lu and X. Ruan, J. Appl. Phys., 2016, 119, 225109.

58 B. Liao, B. Qiu, J. Zhou, S. Huberman, K. Esfarjani and G. Chen, Phys. Rev. Lett., 2015, 114, 115901.

59 M. Bernardi, D. Vigil-Fowler, C. S. Ong, J. B. Neaton and S. G. Louie, Proc. Natl. Acad. Sci. U. S. A., 2015, 112, 5291-5296.

60 R. Forman, W. Brower Jr and H. Parker, Phys. Lett. A, 1971, 36, 395-396.

61 K. Yoshioka and M. Kuwata-Gonokami, Phys. Rev. B: Condens. Matter Mater. Phys., 2006, 73, 081202.

62 P. Scott and C. Jeffries, Phys. Rev., 1962, 127, 32.

63 K. Yoshioka, Y. Morita, K. Fukuoka and M. KuwataGonokami, Phys. Rev. B: Condens. Matter Mater. Phys., 2013, 88, 041201.

64 K. Yoshioka, E. Chae and M. Kuwata-Gonokami, Nat. Commun., 2011, 2, 328.

65 S. Koirala, M. Takahata, Y. Hazama, N. Naka and K. Tanaka, J. Lumin., 2014, 155, 65-69.

66 D. Snoke and G. Kavoulakis, Rep. Prog. Phys., 2014, 77, 116501.

67 S. Mani, J. Jang and J. Ketterson, Phys. Rev. B: Condens. Matter Mater. Phys., 2010, 82, 113203.
68 J. Jang and J. Wolfe, Phys. Rev. B: Condens. Matter Mater. Phys., 2005, 72, 241201.

69 L. Frazer, R. D. Schaller and J. Ketterson, Solid State Commun., 2013, 170, 34-38.

70 A. Mysyrowicz, D. Hulin and A. Antonetti, Phys. Rev. Lett., 1979, 43, 1123.

71 V. So, J. Sipe, M. Fukui and G. Stegeman, J. Phys. C: Solid State Phys., 1981, 14, 4487.

72 V. So, M. Fukui, P. Thomas and G. Stegeman, J. Phys. C: Solid State Phys., 1981, 14, 4505.

73 W. Liu, W. Xie, W. Guo, D. Xu, T. Hu, T. Ma, H. Yuan, Y. Wu, H. Zhao and X. Shen, et al., Phys. Rev. B: Condens. Matter Mater. Phys., 2014, 89, 201201.

74 K. Ishioka, M. Hase, M. Kitajima and H. Petek, Appl. Phys. Lett., 2006, 89, 231916.

75 G. Cho, W. Kütt and H. Kurz, Phys. Rev. Lett., 1990, 65, 764.

76 M. Hase, M. Katsuragawa, A. M. Constantinescu and H. Petek, Nat. Photonics, 2012, 6, 243-247.

77 J. I. Pankove, Optical Processes in Semiconductors, PrenticeHall, Englewood Cliffs, New Jersey, 1971.

78 N. Naka, I. Akimoto and M. Shirai, Phys. Status Solidi B, 2013, 250, 1773-1776.

79 J. P. Wolfe and J. I. Jang, New J. Phys., 2014, 16, 123048.

80 S. Sobkowiak, D. Semkat and H. Stolz, Phys. Rev. B: Condens. Matter Mater. Phys., 2015, 91, 075209. 